\title{
Germanica
}

\section{Der Schleier der Beatrice und das historische Drama}

Beatrice's Veil and historical drama

Le Voile de Béatrice et le drame historique

\section{Wolfgang Sabler}

\section{OpenEdition}

\section{Journals}

Édition électronique

URL : http://journals.openedition.org/germanica/2120

DOI : 10.4000/germanica.2120

ISSN : 2107-0784

Éditeur

Université de Lille

Édition imprimée

Date de publication : 15 juin 2013

Pagination : 53-65

ISBN : 9782913857315

ISSN : 0984-2632

Référence électronique

Wolfgang Sabler, «Der Schleier der Beatrice und das historische Drama », Germanica [Online], 52 | 2013,

Online erschienen am: 20 Juni 2013, abgerufen am 06 Oktober 2020. URL : http://

journals.openedition.org/germanica/2120 ; DOI : https://doi.org/10.4000/germanica.2120

(c) Tous droits réservés 


\title{
Der Schleier der Beatrice und das historische Drama
}

\author{
Wolfgang SABLER \\ Université de Picardie Jules Verne, Amiens
}

Der Schleier der Beatrice gehört zu den vergessenen, ja von der modernen Kritik fast einheitlich verurteilten Dramen Schnitzlers. Von den Stücken Schnitzlers im historischen Kostüm fand nur Der grüne Kakadu Aufnahme in den Kanon der Wiener Moderne. Dass es sich beim Schleier der Beatrice jedoch nicht nur um eine rein konformistische Unterwerfung unter traditionelle Werte der Wiener Gesellschaft um 1900 handelt, sondern um widersprüchliche Versuche zur Erneuerung der Tradition aus dem Geist der Moderne, unterstreicht Konstanze Fliedl:

Seine eigenen historischen Schauspiele gehen vom gründerzeitlichen Kanon aus. Sie sind merkwürdig ambivalente Versuche, ihn zugleich fort- und gegen ihn anzuschreiben. Sie testen immerzu die Möglichkeiten aus, Geschichte wieder als Kontinuität zu begreifen. Aber wenn sie bemüht sind, in der Form Tradition zu wahren und sich in ein literaturhistorisches Kontinuum zu stellen, droht die Gefahr des Epigonalen. Aus dieser Zwickmühle stammen ihre ästhetischen Verrenkungen. Trotzdem sind sie als dramaturgische Experimente zu lesen, Geschichte nicht theatralisch stillzustellen, sondern sie als Objekt und Ursache historischer Erinnerung zu präsentieren ${ }^{1}$. 86 sq.

1. - Konstanze Fliedl : Poetik der Erinnerung, Wien, Köln, Weimar, Böhlau, 1997, 
Das Scheitern der langwierigen Bemühung des Autors um den Schleier der Beatrice ist unzweifelhaft. Doch ermöglicht gerade dieses Scheitern interessante Fragestellungen zur Schnitzlerschen Dramaturgie.

Zwei wesentliche Gründe scheinen Schnitzlers Interesse an Geschichte - oder historischer Verkleidung -, und damit an nicht realistisch intendierten Dramen motiviert zu haben. Als begeisterter Besucher des Burgtheaters wurde Schnitzler in den siebziger und achtziger Jahren des 19. Jahrhunderts im Umfeld des ,gründerzeitlichen Kanons" „dramatisch sozialisiert" 2 . Der Theaterästhetik des Burgtheaters blieb er, jedenfalls als Zuschauer, sein Leben lang treu. In seiner Schauspielkunst sah er die oft einzig würdige Aufführungsstätte für gerade diejenigen seiner Dramen, die er selbst besonders hoch schätzte, wie den Schleier der Beatrice. Andererseits bezeugen zahlreiche Bemerkungen aus seinen Tagebüchern und Briefen, wie sehr seine eigene Wahrnehmung des Theaters im allgemeinen und seiner Werke im Besonderen von einer für die Zeit konstitutiven Spannung zwischen der tatsächlichen Entwicklung des Theaters und einer allgemeinen Erwartungshaltung beeinflusst wurde. In der Tat entstand vielleicht gerade aus der Vorherrschaft - wenn man vom Avantgardedrama absieht - der Konventionen des psychologischen Realismus eine weit verbreitete Erwartung des „großen Dramas“, wie sie in den Theaterschriften und Kritiken der Zeit immer wieder gleichsam als Antwort auf die psychologische Verkleinerung der Welt und die damit einhergehende Abschwächung, wenn nicht Unmöglichkeit, des Tragischen im Theater der Zeit immer wieder beschworen wird. Durchaus charakteristisch dafür ist etwa ein Kommentar Schnitzlers im Tagebuch vom 19. März 1903 zur negativen Kritik Paul Goldmanns die Berliner Aufführung des Schleiers der Beatrice betreffend: „Feuill. Von P.G. über Beatrice; er versucht es als "klein" hinzustellen. - An den wirklichen Dingen gemessen hat er ja recht - aber an dem - was er für gross hält!" 3 . In der Tat hatte Goldmann gerade diesen Aspekt der „Grösse“ hervorgehoben; er war dabei im Übrigen keineswegs der einzige Rezensent:

Schnitzler hat mit seinem Schauspiel [...] ein grosses Drama in der Art der Classiker schaffen wollen. Seine Kräfte haben zur Bewältigung dieser Aufgabe nicht genügt [...]. Es krankt an einem inneren Uebel. Der ganze Apparat eines großen Dramas ist aufgeboten; und doch ist dieses Drama nicht groß - aus dem einzigen und entscheidenden Grunde nicht groß, weil ihm die Größe fehlt ${ }^{4}$.

2. - Ibid.

3. - Arthur Schnitzler :Tagebuch 1903-1908, Wien, Verlag der Österreichischen Akademie der Wissenschaften, 1991.

4. - Neue Freie Presse, 19.3.1903. 
Diese Tautologie ist der Ausdruck der erwähnten für die zeitgenössische Dramaturgie unüberwindbaren Aporie. Es ist, betrachtet man die dramatische Perspektive von Schnitzlers Schaffens, nicht erstaunlich, dass diese „Verkleinerungsphrasen, die über mich stampigliert sind“"5, gerade auf den Psychologen Schnitzler immer wieder angewendet werden, was den Autor sein Leben lang aufs Höchste empört:

Dass man Beatrice, einsamen Weg, Ruf des Lebens geschrieben haben kann... dass man [...] unter den ersten zehn Künstlern einzureihen ist, die heute in deutscher Sprache schreiben - und dass noch in diesem Ton über mich abgeurtheilt werden darf 6 .

\section{Rezeptionsgeschichte}

Der Schleier der Beatrice erlebte seine Uraufführung weder in Wien, noch in Berlin, sondern am 1. Dezember 1900 in Breslau, eine Tatsache, die die schwierige, und für Schnitzler etwas enttäuschende Rezeptionsgeschichte des Stückes veranschaulicht. In Berlin stand Der Schleier erstmals im März 1903 auf dem Programm des Deutschen Theaters von Otto Brahm, auf eine Aufführung im Burgtheater musste Schnitzler bis nach dem Ersten Weltkrieg warten, bis Mai $1925^{7}$.

Während aber die Breslauer Aufführung nur wenig Beachtung fand, bieten die Besprechungen der Berliner Inszenierung von Emil Lessing ein kontrastreiches Spektrum. So enthält Paul Goldmanns bereits erwähnte Rezension in der Neuen Freien Presse charakteristische Topoi ständig propagierter Vorurteile :

Und die Frage, ob es ihm gelingen wird, das hohe Ziel zu erreichen, [...] hängt ab von der Frage, ob er die Kraft haben wird, aus der kleinen und abgesonderten Welt, in der sein Schaffen sich bisher hauptsächlich bewegt hat und in der die Stimmungen - die Stimmungen, die aus den kleinen Gefühlen hervorgehen - eine allzu wichtige Rolle spielen, den Weg zu finden ins große Leben hinein, das allein den Dichter mit jenen starken und tiefen Gefühlen zu erfüllen vermag, aus denen die grossen Werke erwachsen ${ }^{8}$.

Von den meist ziemlich redundanten Rezensionen setzt sich jene von Maximilian Harden in der Zukunft scharf ab, eine analytisch originelle und literarisch hochstehende Auseinandersetzung von außergewöhnlicher Länge :

5. - A. S.: Tagebuch 1903-1908, 21.8.1906.

6. - Ibid., 5.4.1907.

7. - Die Erstaufführung am Burgtheater unter der Regie von Franz Herterich fand am 23. Mai 1925 statt

8. - Neue Freie Presse, 19.3.1903. 
Dieses [...] Schauspiel hat viele Mängel. [...] Dennoch ist das Drama nicht nur das beste, das diesem Dichter gelang: es ragt auch über fast Alles hin, was seit langen Jahren im deutschen Sprachgebiet reifte. [...] In seinem Gedicht [...] ist wirklich Renaissance, nicht nur ihr Kleid ${ }^{9}$.

Schnitzler selbst hat sehr wohl das Stück als „großes Drama“ konzipiert, was auch immer diese Kategorie in seinen Augen bedeuten mochte. Unzweifelhaft hielt er es für bedeutend, worauf er in seinen Tagebuchnotizen immer wieder zurückkommt, auch etwa noch im Dezember 1922 :

Bei allen Schwächen, Affectationen, Epigonismen, ein (nicht grosses, aber in gewissem Sinne bedeutendes) Werk, das vielleicht mehr aufs Theater gehört als irgend eines meiner andern ; - ebensosehr um die Vorzüge ins rechte Licht zu setzen als die Schwächen zu verhüllen ${ }^{10}$.

Das ambitiöse Vorhaben dieses Renaissance-Schauspiels war für Schnitzler Zeichen einer Neuorientierung seiner dramatischen Arbeit. „Mir scheint überhaupt als käme ich jetzt in andere Gegenden"11, schreibt er 1899 an Georg Brandes.

Zugleich war aber Der Schleier der Beatrice ein spezifisch für die schauspielerischen Möglichkeiten am Burgtheater konzipiertes Werk, das in seinen Augen gerade in der Wiederaufarbeitung klassischer Dramen brillierte. Dazu zählte unter anderem das große ShakespeareProjekt unter der Burgtheaterdirektion Schlenther. Als Schnitzler im März 1902 einer Aufführung von Shakespeares Heinrich IV beiwohnte, notierte er in seinem Tagebuch: „Schmerz, dass Bea. nicht an der Burg gespielt wird"12. Auf diese Aufführung sollte Schnitzler ein Vierteljahrhundert warten.

In der Tat hatte Burgtheaterdirektor Schlenther 1900 nach einer anfänglich offensichtlich positiven Reaktion die Aufführung des Stückes abgelehnt. Das führte zwar zu einem Protestschreiben von sechs Intellektuellen in Wiener Tageszeitungen ${ }^{13}$, bedeutete aber für

9. - Maximilian Harden : „Der Schleier der Beatrice“, Die Zukunft, 26.3.1903, S. 529.

10. - A. S. : Tagebuch 1920-1922, Wien, Verlag der Österreichischen Akademie der Wissenschaften, 1993, 26.11.1922.

11. - Brief an Georg Brandes vom 12.1.1899 in : Arthur Schnitzler : Briefe 18751912, Hrsg. Therese Nickl, Heinrich Schnitzler, Frankfurt, S. Fischer 1981, S. 367.

12. - A. S. : Tagebuch 1893-1902, Wien, Verlag der Österreichischen Akademie der Wissenschaften, 1989, 3. 3. 1902.

13. - Die "Erklärung" erschien am 14.9. 1900 in : Neue Freie Presse, Fremdenblatt, Neues Wiener Journal. Dr. Paul Schlenther's Rechtfertigung zum Fall Schnitzler wurde 
Schnitzler einen schweren Rückschlag. In seiner ebenfalls in der Presse veröffentlichten Antwort schreibt Schlenther: „Das Stück und seine dramatische Wirkung rückt mir ferner; und ich möchte fast prophezeien, $\mathrm{da} ß$ Sie nach Jahren, wenn Sie von jüngeren Werken wieder darauf zurückkommen, dieselbe Erfahrung machen werden“. Schlenther kritisiert besonders die Beziehung zwischen dem Herzog und dem Dichter : „Sie sind Begriffe, aus dem theoretischen Contrast geboren. Damit aber ist dem Drama das Rückgrat gebrochen $[\ldots]^{14}$.

Mit seiner Prophezeiung sollte Schlenther nicht recht behalten. Jahrzehntelang wird Schnitzler sich um das Stück bemühen. 1911 notiert er in seinem Tagebuch zu einer Aufführung des Stücks im Hamburger Schauspielhaus:

Hatte sie in den letzten Tagen wieder gelesen, einige Stellen nicht ohne leise Enttäuschung; fand aber manches sehr schön. Das beste dran ist die Beziehung der zwei Männer, Filippo und Herzog - unterirdische, überirdische - und was sie übereinander sagen. Auch die Beatrice als Gestalt wird kaum so bald vergehn ${ }^{15}$.

1912 bedauert er:

Nm. „Beatrice“ gelesen. Zwiespältiger Eindruck. Einige Partien (1. Akt, vieles im 3.) ersten Ranges ; dann manches epigonal, äusserlich shakespearisirend; auch die Verse ungleich [...]. - Das Grundproblem aber von einer Eigenart, die heute kaum noch gespürt wird; und die Beziehung Herzog Dichter von starker wurzeltiefer Bedeutung. Auch die „Beatrice“ hätte Dauerchancen, - die Figur - wenn das Stück eins hätte ${ }^{16}$.

Erst im Umfeld der tatsächlichen Erstaufführung des Schleiers der Beatrice im Burgtheater wird sich Schnitzler von dem Stück distanzieren und von ,teils läppische[r], shakespearisirende[r] und triviale[r] Episodik" sprechen. Als Burgheaterdirektor Herterich 1924 zur Vorbereitung der Beatrice-Aufführung Textkürzungen von ihm verlangt, hält er im Tagebuch fest: „Striche. So viel er will! Wie fremd ist mir diese Renaissancewelt geworden" 17 . Trotzdem urteilt er nach der Generalprobe am 22.5.1925 : „Das Stück recht wirksam, mit manchen

am 15. September 1900 im Neuen Wiener Journal, veröffentlicht.

14. - Dr. Paul Schlenther's Rechtfertigung zum Fall Schnitzler, Neues Wiener Journal, 15. September 1900.

15. - A.S. : Tagebuch 1909-1912, Wien, Verlag der Österreichischen Akademie der Wissenschaften, 1981, 7.11.1911.

16. - Ibid., 28.6.1912.

17. - A.S.: Tagebuch 1923-1926, Wien, Verlag der Österreichischen Akademie der Wissenschaften, 1995, 28.4.1925 (Vgl. dazu auch 3.5. 1924). 
Schönheiten. Einige Scenen von einem irgendwie "classischen Hauch" - trotz vielerlei Dilettantismus und Epigonie“18. Die Aufführung am 23. Mai 1925 ist ein Erfolg, die „Kritiken über Bea. sind im ganzen auffallend gut"19. Das Neue Wiener Tagblatt erinnert in seiner Rezension, wie vor „fünfundzwanzig Jahren“ Schlenther, „der anfangs das Werk aufführen wollte, $[\ldots]$ wortbrüchig“" wurde. Dieses Stück, schreibt der Kritiker, ist „,bei allen berechtigten Einwänden [...] reich an dichterischen Schönheiten [und bekundet] einen Glanz und Schwung der Phantasie wie in keiner anderen Dichtung Schnitzlers"20. Das Neue Wiener Journal beklagt die entstellenden Striche, die ,nicht zuletzt das Entscheidende: die Lust und die Schauer der letzten Nacht vor dem Tod, der draußen lauert" betreffen ${ }^{21}$. Die Neue Freie Presse erklärt das Schicksal des Stücks im Jahre 1900 mit Vorurteilen Schnitzler gegenüber, der auf die Welt der Liebelei eingeengt wurde:

Das Vorurteil sprach dagegen; und da ein Vorurteil [...] ganz besonders in Wien, der Stadt der Vorurteile, unwiderleglich ist [...] so blieb der Schleier der Beatrice durch ein volles Vierteljahrhundert unaufgeführt, obwohl dieses Drama wie kaum ein anderes seines Urhebers nach der Bühne verlangte, für das Theater geschaffen ist ${ }^{22}$.

Diese Rezeptionsgeschichte des Stücks zeigt, dass die Gründe für das mangelnde Theaterglück und die Ablehnung der Beatrice nicht mit den Kriterien der Moderne zusammenhängen. In Wien jedenfalls - in anderen deutschsprachigen Städten ist der Theaterautor Schnitzler um diese Zeit kaum mehr präsent - wird im Jahre 1925 das Schauspiel nicht als gründerzeitlicher Kitsch abgetan.

1900 allerdings verringert die Ablehnung durch das Burgtheater die Aufführungschancen des Stücks. Die Uraufführung findet schließlich am 1. Dezember 1900 im bescheidenen Breslauer Lobe-Theater statt; die Wiener Presse schenkt ihr kaum Beachtung 23 . Schnitzler selbst notiert voll Bitterkeit in seinem Tagebuch: „Abd. Prem. Bea. - Viel Beifall, viel Opposition. - Schlechte Aufführung [...] M.G. [Marie Glümer] weinte im Wagen mit mir und [dem Kritiker Alfred] Kerr über die schlechte Aufführung"24.

18. - Ibid., 22.5.1925.

19. - Ibid., 26.5.1925.

20. - Neues Wiener Tagblatt, 24. Mai 1925.

21. - Neues Wiener Journal, 24.5.1925.

22. - Neue Freie Presse, 24.5.1925.

23. - Im Neuen Wiener Journal vom 2. 12.1900 findet sich eine kurze Rezension.

24. - A.S.: Tagebuch, 1893-1902, 1.12. 1900. 


\section{Der Schleier der Beatrice und die Konkurrenz der Monna Vanna}

Danach hofft Schnitzler auch vergeblich auf eine Aufführung durch Brahm, mit dem er vertraglich verbunden ist. Trotz der insistierenden Nachfragen des Autors wartet Brahm ab und führt schließlich noch vor der Erstaufführung des Schleiers der Beatrice ein anderes Drama mit Renaissancehintergrund auf, Monna Vanna von Maeterlinck, obwohl ihn Schnitzler gebeten hatte, er möge doch auf jeden Fall sein Stück bevorzugen. Hat er sein Stück nicht vor dem Maeterlincks verfasst?25 Schnitzler, der über die begeisterten Rezensionen der französischen Presse informiert gewesen zu sein scheint, befürchtet zu Recht, dass ihm der Erfolg der Monna Vanna schaden würde. In der Tat erzielt dieses Renaissancedrama nach seiner Premiere am Deutschen Theater am 24. November 1902 einen überwältigenden Erfolg und wird mehr als tausend Mal auf deutschsprachigen Bühnen aufgeführt.

Die Berliner Erstaufführung des Schleiers der Beatrice am 7. März 1903 sollte Schnitzlers Befürchtungen dann auch bestätigten. Trotz seiner äußerst konfliktreichen Interventionspolitik in die Regie Emil Lessings, wobei es unter anderem um die schwierige Szene der allgemeinen Orgie in Akt IV geht, für die Lessing in seiner realistischen Theaterkonzeption nicht viel übrig hat, hat er nach der Aufführung das „Gefühl eines gelinden Durchfalls auf der Bühne“26. Die Kritiken fallen meist negativ aus. Noch Jahre danach wird Schnitzler in einem Brief Hermann Bahr zu dessen Einakter Der Faun den Ratschlag geben, im Falle einer Aufführung ,ihn nicht von Lessing inszenieren zu lassen, der Orgien nur ein mäßiges Verständnis entgegenbringt, was sich im vierten Akt der Beatrice jammervoll erwies“ ${ }^{* 27}$.

In der Tat stellt ein Stück wie Beatrice an ein realistisch geprägtes, eher am Naturalismus orientiertes Theaterkonzept wie das Brahms, unüberwindliche Anforderungen. Schnitzler kennt die Grenzen dieser Dramaturgie, hat aber nicht Mut genug, daraus die notwendigen Schlüsse zu ziehen, obwohl er dazu eine außergewöhnlich interessante Möglichkeit gehabt hätte. In der Tat erhält er am 31.8.1902 einen Brief eines jungen und aufstrebenden Theatermannes: Max Reinhardt. Reinhardt schreibt ,[...] doch glaube ich, daß Ihr Stück derzeit an keiner anderen Bühne so gespielt, inszeniert und ausgestaltet werden

25. - Vgl. A.S.: Tagebuch, 1893-1902, 24. 11. 1902.

Maurice Maeterlinck : Monna Vanna. Pièce en trois actes, Paris, Charpentier et Fasquelle, 1902 ; deutsche Übersetzung : Monna Vanna. Schauspiel 3 Afz, dt. Friedr. v. Oppeln-Bronikowski, autorisirte Ausgabe, Leipzig, Eugen Diederichs, 1903.

26. - A.S.: Tagebuch, 1903-1908, 7.3. 1903.

27. - Brief an Hermann Bahr vom 24.6.1906. Briefe 1875-1912, S. 537. 
kann wie auf der unseren“. Gerade sein Kleines Theater (früher Schall und Rauch) sei fähig einem Versdrama Theaterexistenz zu geben.

Vornehmlich aber glaube ich, daß das Versdrama, bei uns von dem Schall und Rauch großer Ausstattungstheater befreit, wirklich lebendig werden kann, umso mehr als durch den Umbau unserer Bühne ein Apparat geschaffen wurde, der die feinsten, subtilsten und neuesten Wirkungen ermöglicht ${ }^{28}$.

Doch verweigert ihm Schnitzler das Stück. Dieses Fehlurteil hat wohl das Verhältnis zu Reinhardt von Anfang an belastet, das im Weiteren von ständigem Missverständnis behaftet bleiben wird. Was Schnitzler nicht verstanden hat, war die neuaufstrebende Rolle des Regisseurs. Kaum ein anderer wie Max Reinhardt vertritt diese neue fundamentale Entwicklung des Theaters, das sich vom traditionellen Texttheater tendenziell entfernt und dem Regisseur die zentrale Rolle im Theaterschaffen gibt, wobei wohl der Autor - und der Text - in den Hintergrund tritt gegenüber dem vom Regisseur geschaffenen Theaterkunstwerk. Dieser Entwicklung stand Schnitzler relativ verständnislos gegenüber. Seine Verweigerung des Angebots von Max Reinhardt ist also auch Ausdruck der vormodernen auktorialen Theaterkonzeption Schnitzlers. Das Regietheater Reinhardts hätte dem Stück durchaus ästhetischen Erfolg verschaffen können; aber dazu hätte er es Reinhardt zur freien Verwendung überlassen müssen.

Die realistische Theaterkonzeption Brahms entspricht den damals auf den großen Bühnen vorherrschenden Prinzipien. In dieser Sicht des Theaters, die der Wahrscheinlichkeit und der Lebensnähe große Bedeutung beimisst, sollte jeder theatralische Effekt vermieden werden, was zum Beispiel defacto durch die postulierte Notwendigkeit nicht-theatralischer, das heißt im Wesentlichen dem Alltäglichen nachgeahmten Körperhaltungen und Dialoghaltungen, den Ausdrucksmöglichkeiten der Schauspieler aber auch der Dramenhandlung selbst relativ enge Grenzen setzte.

Die Wahl eines historischen Zeitpunkts für die Handlung erlaubte in diesem Sinne den allzu strengen Regeln dieses angeblich lebensnahen Realismus zu entkommen. In der Tat scheinen diese ungeschriebenen Konventionen vor allem für die Dramen gegolten zu haben, deren Handlung in der „Gegenwart“ spielt. Der Schleier der Beatrice bemächtigt sich mit Genuss dieser Entgrenzung. Die Wahl des Verses ermöglicht, die Künstlichkeit des Spiels zu unterstreichen. Das erste Aparté befindet sich gleich am Beginn des Stücks, der erste Monolog

28. - Der Briefwechsel Arthur Schnitzlers mit Max Reinhardt und dessen Mitarbeitern, Hrsg. Renate Wagner, Salzburg, Otto Müller Verlag, 1971, S. 41-42. 
(Filippos) leitet den ersten Szenenwechsel ein. Im realistisch-illusionistischen Theater sind sie streng verpönt. Kuss und Kampf (angedeutet durch Degenziehen) wie bei Romeo und Julia scheinen hier durch ihre Relegierung in die Vergangenheit gleichsam natürlich. Man schwächt das Gewicht realistischer Konventionen ab und die Handlungen können vom gemeinhin Erwarteten abweichen. Ein kurioser Artikel zu Monna Vanna aus dem Neuen Wiener Tagblatt unterstreicht den zeitgemäßen und offensichtlich breit akzeptierten Charakter dieser Fragestellungen. Der offensichtlich rechtskundige Verfasser des Artikels mit dem Titel Monna Vanna vor dem Strafgesetze stellt eingangs fest: „Der Realismus in der dramatischen Literatur ist noch lange kein vollständiger; immer noch spielen sich Dinge auf der Szene ganz anders ab, als in der natürlichen Wirklichkeit." Der Artikel enthält dann eine (ungewollt) komische Aufzählung aller Gesetzesübertretungen in Monna Vanna, für die jeweils die Höhe der normal vorgesehenen Geldstrafen fixiert wird und schließt mit den Worten: "Wie herrlich schön sind doch die Sphären, in denen die Dichter wandeln, wo es kein Strafgesetz gibt und keinen Sicherheitswachmann!" 29

\section{Renaissance-Dramen... und die Wiederentdeckung des Körpers}

Hinter dem augenscheinlichen Traditionalismus beider RenaissanceStücke verbirgt sich in der Tat ein Bruch mit dem gemeinen Theatergesetz. Allerdings bewegen sich beide Autoren in gewisser Weise in gegensätzlicher Richtung: Maeterlinck tritt aus der symbolistischen Theaterwelt in eine mehr den Regeln des üblichen Theaterbetriebs entsprechende. Paul Goldmann analysiert Monna Vanna in seiner Rezension von beeindruckender Länge als eine Rückkehr in die wahre Welt des Theaters ${ }^{30}$. Schnitzler hingegen verlässt den Rahmen seiner von der Kritik häufig als klein erachteten Welt psychologisch realistischer Beobachtung und Verhaltenskritik und erobert sich in der historischen Dimension neue Freiräume.

Das Unwahrscheinliche ist hier erlaubt. Weibliche Untreue und Ehebruch sind ein obsessiv behandeltes Thema der Theaterproduktion der Zeit. Im Theateruniversum der Gegenwart kann daraus kein positives moralisch akzeptables Ende gesponnen werden. Doch wenn Monna Vanna, die Frau des Kommandanten der Festung Pisa, sich für ihre Stadt aufopfert, als Prinzivalle, der Anführer der florentinischen Belagerer, als Bedingung für die Aufhebung der Besatzung und die Belieferung der hungernden Stadt mit Lebensmitteln fordert, dass

29. - Neues Wiener Tagblatt, 8.3. 1903.

30. - Neue Freie Presse, 15.10.1902. 
sie sich, nur mit einem Mantel bekleidet, in sein Zelt begeben solle, wird ein im Gegenwartsdrama undenkbarer Kontext geschaffen und dann weiterentwickelt. In der Tat liebt Prinzivalle Monna Vanna seit seiner Jugend. In der fatalen Nacht lässt er sie unberührt und begibt sich mit ihr in die Stadt, da er nun in Florenz als Verräter gilt. Doch die - allerdings den ungeschriebenen Regeln der realistisch-gegenwärtigen Theaterwelt entnommene - Unfähigkeit des Kommandanten, seiner Frau zu glauben, führt schließlich dazu, dass letztere mit Prinzivalle flieht, den sie nun liebt. Durch diese dialektische Umkehrung üblicher Theaterverhältnisse erscheint nun Monna Vanna weiterhin so „rein“ wie sie anfangs gepriesen wurde, obwohl sie ihre Ehe bricht, ja dieser Ehebruch erscheint als moralisch notwendig. Solche Problemstellungen mögen von heute aus gesehen abstrus wirken, Tatsache ist, dass hier die historische Situierung Freiheiten erlaubt, die im Rahmen der sonst allgemein gültigen Konventionen undenkbar sind.

Bei Schnitzler widerspricht ganz besonders der vierte Akt allen üblichen Regeln, ja überschreitet eindeutig die Darstellungshorizonte des realistischen Theaters. Bologna wird vom Papst belagert und erlebt seine letzte Nacht vor dem Untergang. In dieser Nacht vor dem Ende der bestehenden Gesellschaftsordnung brechen plötzlich alle Regeln auf, werden die Gesetze ungültig. Als in dieser Nacht der Herzog ein Bürgermädchen begehrt, heiratet er es sofort, als letzteres diese Bedingung stellt, um ihm zu folgen. In der realistischen Theaterwelt, die in der Gegenwart angesiedelt ist, sind große soziale Unterschiede hingegen ausnahmslos unüberwindbar. Doch stellt das nur den Anfang dar. Der Herzog öffnet zur Feier des Tages seinen Garten allen Einwohnern Bolognas mit der expliziten Aufforderung, sich dort zu vergnügen, wie es ihnen gefalle.

Ihr andern, nützt die Zeit! Nehmt meinen Garten

Als duftend Lager eurer Freuden hin!

[...] Ich aber, euer Fürst,

Jeglichem Bund, der heut nacht sich schließt, Geb' ich die Weihe ${ }^{31}$.

Dass gerade diese Analyse der allgemeinen Orgie als Ausdruck des Zerfalls jeglicher Ordnung im Mittelpunkt des Stücks und des Interesses der Kritiker steht, die sich mit dem Stück ernsthaft beschäftigt haben, zeigt unter anderem der Beginn der Besprechung der Beatrice durch Harden:

31. - Arthur Schnitzler: Der Schleier der Beatrice, in : Die dramatischen Werke, Frankfurt, S. Fischer, 1962, Bd. 2, S. 654. 
Durch Schloß und Garten des Herzogs von Bologna rast trunkene Gier. Lüste suchen, Lüste finden, umschlingen einander und auf der Wiese, die sich hinter Terrasse und Garten dehnt, vermählt das Gestöhn der lechzenden und der satten Paare sich zu einem langen, pausenlosen Brunstseufzen, dessen Anhauch die Fackeln zusammenzucken und wieder aufflackern läßt [...]. Was sonst verboten ist, ist heute erlaubt. $[\ldots]^{32}$.

Schnitzler hat dieses Thema immer wieder aufgegriffen (Komödie der Verführung, Hirtenflöte). Der Schleier der Beatrice dramatisiert die vielfältigen Brüche der gesellschaftlichen Ordnung im individuellen (man denke z.B. an Filippos plötzliche unverständliche Abwendung von allem, was bisher seine Person ausgemacht hat), wie auch im gesellschaftlichen Rahmen (z.B. die Haltung des Herzogs und des Hofs), die unweigerlich zur Katastrophe führen müssen, aber auch von der erwarteten Katastrophe des Untergangs Bolognas beschleunigt werden.

Eine derartige Analyse extremer Situationen und die sich daraus ergebende Radikalisierung der Haltungen sind im Theater um 1900 nur in der Rückversetzung in die Geschichte möglich. Die Renaissance dient dabei als entsprechende Kulisse, da die gezeigten Vorgänge den am Ende des 19. Jahrhunderts geläufigen, von Burckhardt stark beeinflussten Vorstellungen dieser Epoche entsprechen. Schnitzlers Stück entstand im Übrigen zu Beginn einer wahren Renaissance-Mode im Theater $^{33}$. Dabei geht es weder Schnitzler noch Maeterlinck um historische Genauigkeit.

Tatsächlich wurde Bologna 1506 von Papst Julius II. annektiert und die Familie Bentivoglio aus Bologna verjagt, wenn auch der bis dahin die Stadt beherrschende Herzog nicht den Namen Leonardo, sondern Giovanni II trug. Allerdings konnte der Papst im Gegensatz zur Darstellung im Schleier, die Bologna geeint in patriotischem Widerstand gegen ihn zeigt, die Stadt gerade aufgrund der dort herrschenden inneren Konflikte erobern. Ein Aufstand gegen den Herzog wurde wenige Jahre davor niedergeschlagen. Er wurde angeführt von einem gewissen Mariscotti, der im Drama zum Verräter wird. Diese Vereinfachungen braucht Schnitzler, um die Endzeitstimmung glaubhaft zu machen, die im wahren historischen Kontext weniger fundiert gewesen wäre. Die Künstlerpersonen sind erfunden, entsprechen aber den gängigen Vorstellungen zur Künstlerwelt in der Renaissance, deren

32. - Maximilian Harden : „Der Schleier der Beatrice“, Die Zukunft, 28.3.1903, S. $517-530$.

33. - Gerd Uekermann : Renaissancismus und Fin de siècle. Die italienische Renaissance in der deutschen Dramatik der letzten Jahrhundertwende, Berlin, de Gruyter, 1985. 
intellektuelles Umfeld durch diverses, mehr oder minder anekdotisches Beiwerk ausgemalt wird.

Maeterlincks Drama spielt fast genau zum gleichen Zeitpunkt, am Ende des 15. Jahrhunderts. Die Rivalität zwischen Florenz und anderen Stadtstaaten ist zwar geschichtlich, doch es existierte kein Söldnerführer namens Prinzivalle. Ein gewisser Prinzivalle della Stuffa nahm offensichtlich an einer von Papst Julius II. inspirierten Verschwörung zu Beginn des 16. Jahrhunderts teil, bei der die Florentiner Führung hätte ermordert werden sollen. Prinzivalle musste dann nach Siena fliehen ${ }^{34}$. Pisa spielt seinerseits in dem Kampf um die Macht in Florenz eine wichtige Rolle und wird tatsächlich 1509 belagert und schließlich erobert.

Der geschichtliche Kontext wird also durchaus nachempfunden, die Einzelheiten werden aber je nach den dichterischen Bedürfnissen frei erfunden. Dies ist etwa auch der Fall mit einem dritten Renaissancedrama der Zeit, Novella d'Andrea von Ludwig Fulda, das die Geschichte der gleichnamigen Juristin erzählt, die in der ersten Hälfte des 14. Jahrhunderts in Bologna gelehrt hat, allerdings in einer für die Bedürfnisse der Diskussion um die Emanzipation der Frauen in der Zeit um 1900 zurechtgerückten Perspektive, bei der die Berufsausübung als Universitätsprofessorin, entgegen der historischen Wahrheit, als eine Liebe und Ehe ausschließende Wahl dargestellt wird ${ }^{35}$.

Die in diesem Phantasieumfeld von den üblichen realistischen Konventionen befreite Handlung erlaubt bei Schnitzler auch eine Erneuerung der Darstellungsweise und besonders eine Befreiung des Körpers der Schauspieler von allzu engen Verhaltensregeln:

Der Saal wird leer. auch dunkler; einige Lichter verlöschen; die Fackeln im Garten immer unruhiger, düsterer; aus der Wiese undeutlich wahrnehmbare Bewegung; Paare gleiten vorüber, umarmen sich, sinken hin, doch Alles wirkt wie Schattenbilder; manchmal stürzen Frauen wie fliehend vorbei ${ }^{36}$.

Das Theater der Zeit zeichnet sich durch eine langsame Rückkehr des Körpers auf die Bühne aus, wie sie sich unter anderem in Szenen wie der eben zitierten zeigt. Solche Szenen nehmen Distanz zu der sehr zurückhaltenden Einsetzung des Körpers im Spiel, die in unzähligen Nebenbemerkungen in den Dramen der Zeit dokumentiert ist. Die weitgehend zurïckgedrängten Theatertraditionen mit intensiver physischer

34. - Götz-Rüdiger Tewes : Kampf um Florenz-Die Medici im Exil (1494-1512), Köln u.a., Böhlau, 2011, S. 884 sqq.

35. - Ludwig Fulda : Novella d'Andrea, Schauspiel in 4 Aufzügen, Stuttgart und Berlin, 1904.

36. - Arthur Schnitzler, Der Schleier der Beatrice, in : Die dramatischen Werke, Bd. 2, S. 654. 
Präsenz der Schauspieler, wie die Comedia dell'arte, erleben nun eine Wiedergeburt. Die dramatische Literatur ist überreich an Versuchen verschiedenster Autoren auf diesem Gebiet, darunter Schnitzler (Die Verwandlungen des Pierrot). Es handelt sich unter anderem um Versuche, dem Theater neue Territorien zu erobern, seine Grenzen zu erweitern. Damit hängt auch ein immer größeres Interessen an nichttextuellen dramatischen Formen zusammen, wie etwa der Pantomime oder Marionettenspielen. Diese bemerkenswerte Beschäftigung mit neuen Formen hat weniger mit der oft dazu herbeizitierten Sprachkrise zu tun, als mit einer fundamentalen Entwicklung des Theaters, das sich, in der Perspektive der Gesamtproduktion, tendenziell vom Texttheater $\mathrm{zu}$ befreien sucht und neue Grenzlinien für sich zu ziehen beginnt, als eigene, nicht nur textinterpretierende Kunstform. Dass Schnitzler den Schleier der Beatrice von Anfang an in verschiedenen Varianten konzipiert hat, ist bekannt ${ }^{37}$. Der Schleier der Pierrrette, als im 19. Jahrhundert spielende Pantomime und Der Schleier der Beatrice, als historisches Drama, nehmen Anteil an dieser künstlerischen Öffnung des Theaterhorizonts. 
